\title{
Total Variance Approach on Commercial Vehicle Handling Dynamics
}

\author{
Yaohua GUO and Jinquan DING \\ Yutong Bus Co., Ltd, Zhengzhou, China
}

\begin{abstract}
The suspension roll motion can produce roll steer, which are functions of roll angle, thus producing extra lateral forces. This paper develops the total variance approach to analyze the effect of suspension roll on commercial vehicle handling dynamics. The side-slip angle unified transfer function, reaction time, transition time, damping ratio and total variance are introduced with the effect of suspension. The vehicle designers could use this approach to get optimization design parameters of vehicle without numerical calculation. For the two-axle commercial vehicles, the total variance approach is useful and could serve as an important tool for evaluating the effect of vehicle suspension roll on commercial vehicle dynamics.
\end{abstract}

Keywords. Suspension roll steer, total variance approach, side-slip angle unified transfer function, vehicle handling dynamics

\section{Introduction}

The suspension roll steer and camber thrust are import factor for vehicle handling characteristics. The usual evaluation indexes could only represent single performance, it needs to evaluate the comprehensive performance by using one comprehensive vehicle handling characteristics evaluation index.

Schunck and Riekert established the linear two degree freedom model of vehicle dynamics [1]. Segel [2] established the three degree freedom model vehicle dynamics. Abe [3] presented an equivalent approach to analyze the effect of suspension roll on two-axle vehicle. K. Guo [4] analyzed the natural frequency, damping ratio, reaction time, transition time and total variance of the two-axle vehicle. Ding J [5] presented an equivalent approach to analyze both the effect of body roll and n-axle handling on vehicle dynamics by the equivalent wheelbase and steady factor. Y. Zhao [6] studied the total variance approach for the vehicle closed-Loop control System. K. Guo [7] justified the use of total variance analysis approach.

This paper develops the total variance approach to analyze the effect of suspension on commercial vehicle handling dynamics. For the two-axle commercial vehicle, the

\footnotetext{
${ }^{1}$ Corresponding Author, Yutong Bus Co., Ltd, Zhengzhou 450004, China; E-mail:dingjqxs@163.com
} 
mathematical handling concepts, such as side-slip angle unified transfer function, reaction time, transition time, damping ratio and total variance, are calculated to analyze the effect of suspension roll steer without numerical calculation. The vehicle designers could use this approach to get optimization design parameters of vehicle.

\section{Commercial vehicle model inclusive of roll}

According to Ding J [5], the equations 3DOF can be converted to

$$
\begin{aligned}
& M V \dot{\beta}+2\left(K_{1}+K_{2}\right) \beta+\left[M V+\frac{2\left(x_{1} K_{1}+x_{2} K_{2}\right)}{V}\right] r-M_{s} h \dot{p}-2 Y_{\phi} \phi=2 K_{1} \delta \\
& 2\left(x_{1} K_{1}+x_{2} K_{2}\right) \beta+I_{z} \dot{r}+\frac{2\left(x_{1}^{2} K_{1}+x_{2}^{2} K_{2}\right)}{V} r-I_{x z} \dot{p}-2 N_{\phi} \phi=2 x_{1} K_{1} \delta \\
& -M_{s} h V \dot{\beta}-I_{x z} \dot{r}-M_{s} h V r+I_{\phi} \dot{p}+C_{\phi} p+\left(K_{\phi}-M_{s} g h\right) \phi=0
\end{aligned}
$$

where

$$
\begin{gathered}
Y_{\phi}=\left(\frac{\partial \alpha_{1}}{\partial \phi} K_{1}+\frac{\partial \alpha_{2}}{\partial \phi} K_{2}\right)-\left(K_{c 1} \frac{\partial \phi_{1}}{\partial \phi}+K_{c 2} \frac{\partial \phi_{2}}{\partial \phi}\right) \\
N_{\phi}=\left(\frac{\partial \alpha_{1}}{\partial \phi} x_{1} K_{1}+\frac{\partial \alpha_{2}}{\partial \phi} x_{2} K_{2}\right)-\left(K_{c 1} x_{1} \frac{\partial \phi_{1}}{\partial \phi}+K_{c 2} x_{2} \frac{\partial \phi_{2}}{\partial \phi}\right)
\end{gathered}
$$

By assuming the steady-state roll angle as

$$
\phi=\left(M_{s} h V r+M_{s} h V \dot{\beta}+I_{x z} \dot{r}-I_{\phi} \dot{p}-C_{\phi} p\right) /\left(K_{\phi}-M_{s} g h\right) \approx \frac{M_{s} h V r}{\left(K_{\phi}-M_{s} g h\right)}
$$

The equivalent 2DOF equations of motion with roll effect can be expressed as follows.

$$
\begin{aligned}
& M V(\dot{\beta}+r)=2 K_{1}\left[\delta-\beta-\frac{x_{1}^{\prime}}{V} r\right]+2 K_{2}\left[-\beta-\frac{x_{2}^{\prime}}{V} r\right] \\
& I_{z} \dot{r}=2 K_{1} x_{1}\left[\delta-\beta-\frac{x_{1}^{\prime}}{V} r\right]+2 K_{2} x_{2}\left[-\beta-\frac{x_{2}^{\prime}}{V} r\right]
\end{aligned}
$$

where

$$
x_{i}^{\prime}=x_{i}\left(1+E_{i} V^{2}\right)
$$




$$
E_{i}=-\frac{M_{s} h\left(\frac{\partial \alpha_{i}}{\partial \phi}-\frac{K_{c i}}{K_{i}} \frac{\partial \gamma_{i}}{\partial \phi}\right)}{x_{i}\left(K_{\phi}-M_{s} g h\right)}
$$

The side-slip angle and yaw rate unified transfer function were

$$
\begin{aligned}
& \frac{\beta}{\delta}(s)=Z_{\beta} \frac{\tau_{1}^{\prime} s+1}{Z_{2} s^{2}+Z_{1} s+1} \\
& \frac{r}{\delta}(s)=Z_{r} \frac{\tau_{1} s+1}{Z_{2} s^{2}+Z_{1} s+1}
\end{aligned}
$$

where, $Z_{\beta}$ is the steady-state side-slip angle gain, $Z_{r}$ is the steady-state yaw rate gain.

The steady-state side-slip angle gain $Z_{\beta}$ for two-axle vehicle is

$$
\begin{aligned}
& Z_{\beta}=\frac{\beta}{\delta}(s)_{\substack{\mid s=0 \\
n=2}}=\frac{\frac{4 K_{1} K_{2} x_{2}^{\prime}\left(x_{2}-x_{1}\right)}{V}-2 x_{1} K_{1} M V}{-2 M V\left(K_{1} x_{1}+K_{2} x_{2}\right)+\frac{4 K_{1} K_{2}\left(x_{1}-x_{2}\right)\left(x_{1}^{\prime}-x_{2}^{\prime}\right)}{V}} \\
& \tau_{1}^{\prime}=\frac{2 K_{1} I_{z}}{\frac{4 K_{1} K_{2} x_{2}^{\prime}\left(x_{2}-x_{1}\right)}{V}-2 x_{1} K_{1} M V} \\
& Z_{1}=\frac{\left[I_{z}\left(K_{1}+K_{2}\right)+M\left(x_{1} x_{1}^{\prime} K_{1}+x_{2} x_{2}^{\prime} K_{2}\right)\right] V}{2\left[\left(K_{1}+K_{2}\right)\left(x_{1} x_{1}^{\prime} K_{1}+x_{2} x_{2}^{\prime} K_{2}\right)-\left(x_{1} K_{1}+x_{2} K_{2}\right)\left(x_{1}^{\prime} K_{1}+x_{2}^{\prime} K_{2}\right)\right]-M\left(x_{1} K_{1}+x_{2} K_{2}\right) V^{2}} \\
& Z_{2}=\frac{M I_{z} V^{2}}{4\left[\left(K_{1}+K_{2}\right)\left(x_{1} x_{1}^{\prime} K_{1}+x_{2} x_{2}^{\prime} K_{2}\right)-\left(x_{1} K_{1}+x_{2} K_{2}\right)\left(x_{1}^{\prime} K_{1}+x_{2}^{\prime} K_{2}\right)\right]-2 M\left(x_{1} K_{1}+x_{2} K_{2}\right) V^{2}}
\end{aligned}
$$

\section{Effect of suspension roll on two-axle Commercial vehicle dynamics}

The effect of suspension roll on two-axle vehicle was compared and analyzed with suspension roll steer values $(\mathrm{A} / \mathrm{B} / \mathrm{C} / \mathrm{D})$ in Table 1 as follows. 
Table 1. Suspension roll steer values

\begin{tabular}{ccc}
\hline & $\partial \alpha_{1} / \partial \phi$ & $\partial \alpha_{2} / \partial \phi$ \\
\hline A & 0 & 0 \\
B & -0.121 & 0.031 \\
C & -0.341 & 0.031 \\
D & -0.341 & 0.131 \\
\hline
\end{tabular}

(1) The turning radius ratio inclusive of roll steer effect is increased, and the increasing is more with the velocity increasing. By sign convention, positive roll steer value is an oversteering effect on the front axle and an understeering effect on the second axle. Figure 1 shows the relationship between the turning radius ratio and the travelling speed with/without influence of suspension roll steer.

$$
R / R_{0}=\left(1+U_{2} V^{2}\right)
$$

where

$$
U_{2}=\frac{2\left[\left(K_{1}+K_{2}\right)\left(x_{1}^{2} E_{1} K_{1}+x_{2}^{2} E_{2} K_{2}\right)-\left(x_{1} K_{1}+x_{2} K_{2}\right)\left(x_{1} E_{1} K_{1}+x_{2} E_{2} K_{2}\right)\right]-M\left(x_{1} K_{1}+x_{2} K_{2}\right)}{2 K_{1} K_{2}\left(x_{1}-x_{2}\right)^{2}}
$$

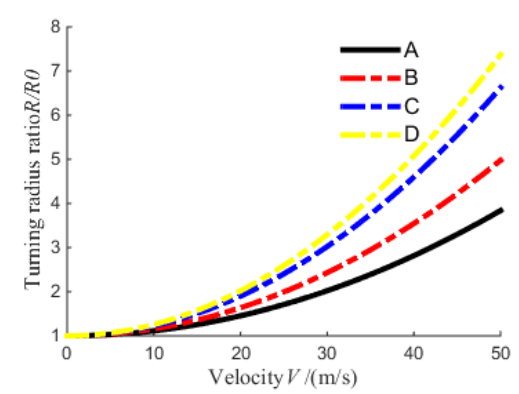

Figure 1. Relation of $R / R_{0}$ and $V$ for the two-axle vehicle

The reaction time $Z_{f}$ was shown in Figure 2 with the effect of vehicle suspension roll. Positive roll steer at the second suspension, or negative roll steer at the front suspension, could reduce the reaction time.

$$
Z_{f}=\frac{I_{z} V\left[x_{1} K_{2}-x_{2} K_{2}\right]}{2 x_{1}\left[\left(K_{1}+K_{2}\right)\left(x_{1} x_{1}^{\prime} K_{1}+x_{2} x_{2}^{\prime} K_{2}\right)-\left(x_{1} K_{1}+x_{2} K_{2}\right)\left(x_{1}^{\prime} K_{1}+x_{2}^{\prime} K_{2}\right)\right]-M x_{1}\left(x_{1} K_{1}+x_{2} K_{2}\right) V^{2}}
$$

The transition time $Z_{c}$ was shown in Figure 3 with the effect of vehicle suspension roll. Positive roll steer at the second suspension, or negative roll steer at the front suspension, could reduce the transition time. The effect of higher values of roll steer at the second suspension lead to decrease the transition time after reaching certain travelling speed.

$$
Z_{c}=\frac{M I_{z} V}{\left[I_{z}\left(K_{1}+K_{2}\right)+M\left(x_{1} x_{1}^{\prime} K_{1}+x_{2} x_{2}^{\prime} K_{2}\right)\right]}
$$




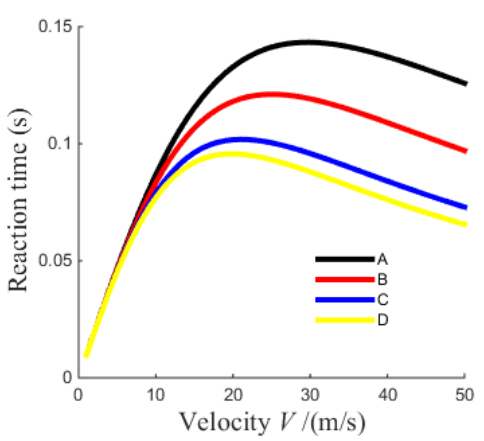

Figure 2. Relation of reaction time $Z_{f}$ and $V$

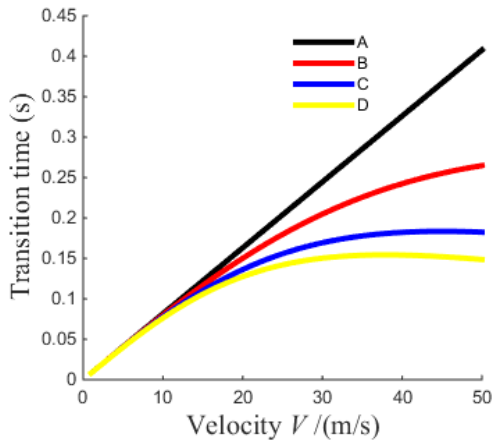

Figure 3. Relation of transition time $Z_{c}$ and $V$

(2)The damping ratio $\xi$ was shown in Figure 4, which was increased with positive roll steer at the second suspension, or negative roll steer at the front suspension. The configurations $\mathrm{C}$ and $\mathrm{D}$ show higher values of roll steer at the second suspension lead to increase the damping ratio after reaching certain travelling speed.

$$
\begin{aligned}
& \xi=\frac{\left[I_{z}\left(K_{1}+K_{2}\right)+M\left(x_{1} x_{1}^{\prime} K_{1}+x_{2} x_{2}^{\prime} K_{2}\right)\right] V}{2\left[\left(K_{1}+K_{2}\right)\left(x_{1} x_{1}^{\prime} K_{1}+x_{2} x_{2}^{\prime} K_{2}\right)-\left(x_{1} K_{1}+x_{2} K_{2}\right)\left(x_{1}^{\prime} K_{1}+x_{2}^{\prime} K_{2}\right)\right]-M\left(x_{1} K_{1}+x_{2} K_{2}\right) V^{2}} \\
& /\left(\sqrt{\frac{M I_{z} V^{2}}{4\left[\left(K_{1}+K_{2}\right)\left(x_{1} x_{1}^{\prime} K_{1}+x_{2} x_{2}^{\prime} K_{2}\right)-\left(x_{1} K_{1}+x_{2} K_{2}\right)\left(x_{1}^{\prime} K_{1}+x_{2}^{\prime} K_{2}\right)\right]-2 M\left(x_{1} K_{1}+x_{2} K_{2}\right) V^{2}}}\right)
\end{aligned}
$$

(3)According to the K. Guo [7], the calculated total variance equation for the two-axle vehicle is

$$
E_{\delta}=\frac{Z_{2}+\left(Z_{1}-\tau_{1}\right)^{2}}{2 Z_{1}}
$$

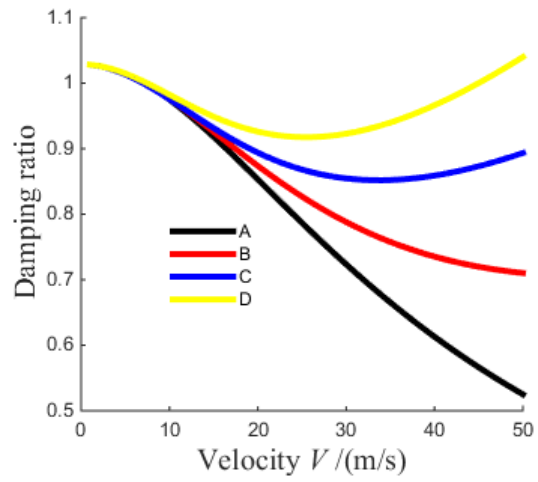

Figure 4. Relation of damping ratio $\xi$ and $V$

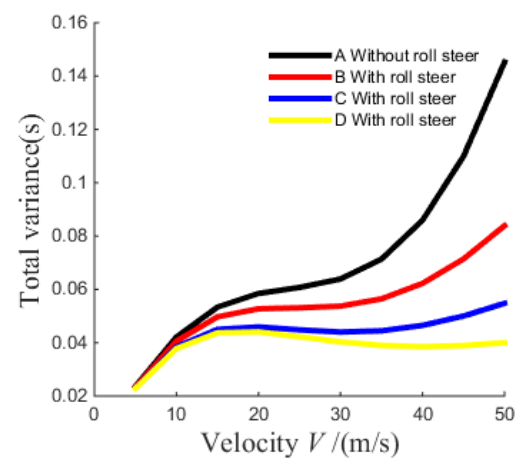

Figure 5. Total variance for the two-axle vehicle

Figure 5 shows the relationship between the total variance and the travelling speed with/without influence of suspension roll steer. Positive roll steer at the second suspension, or negative roll steer at the front suspension, could reduce the total 
variance. So the vehicle response degree following the step steer input is better. According to equation(3.6), it could get the optimization design parameters for the twoaxle vehicle by minimizing the total variance $E_{\delta}$.

\section{Conclusions}

In conclusion, with positive roll steer at the second suspension, or negative roll steer at the first suspension, the effect of suspension roll was analyzed. The turning radius ratio inclusive of roll steer effect is increased, and the increasing is more with the velocity increasing, changing the vehicle steer characteristics toward understeer. The reaction time and transition time are reduced with the effect of vehicle suspension roll. The damping ratio is increased with the effect of vehicle suspension roll. Positive roll steer at the second suspension, or negative roll steer at the front suspension, could reduce the total variance. The vehicle response degree following the step steer input is better. It could get the optimization design parameters for the two-axle vehicle by minimizing the total variance.

\section{References}

[1] Riekert P, Schunck T E. Zur Fahrmechanik des gummibereiften Kraftfahrzeugs[J]. Archive of Applied Mechanics, 1941, 12(1):70-70.

[2] Segel L. Theoretical prediction and experimental substantiation of the response of the automobile to steering control[J]. Proceedings of the Institution of Mechanical Engineers: Automobile Division, 1956, 10(1): 310-330.

[3] Masato Abe, Vehicle Handling Dynamics Theory and Application, Japan (2009), pp. 166-196.

[4] K. Guo, Vehicle Handling Dynamics, China (1991), pp.245-251.

[5] Ding J, Guo K. Development of a generalised equivalent estimation approach for multi-axle vehicle handling dynamics[J]. Vehicle System Dynamics, 2016:1-38.

[6]Youqun Zhao, Guangde Zou, Baohu Liu. Active Safety Index and Calculation Method of Man-Vehicle Road Closed-Loop Control System[J]. Journal of Shandong University of Technology (Natural Science Edition), 1997(2): 7-10.

[7] K. Guo, D. Wang. Research on evaluation method of vehicle handling stability test at high speed[J]. Automobile Technology, 1980(02):21-30. 